\title{
Novel Polyvinyl Alcohol/Styrene Butadiene Rubber Latex/Carboxymethyl Cellulose Nanocomposites Reinforced with Modified Halloysite Nanotubes
}

\author{
Yanjun Tang, ${ }^{1,2}$ Dingding Zhou, ${ }^{1}$ and Junhua Zhang ${ }^{3}$ \\ ${ }^{1}$ Key Laboratory of Advanced Textile Materials and Manufacturing Technology, Ministry of Education, Zhejiang Sci-Tech University, \\ Hangzhou 310018, China \\ ${ }^{2}$ Tianjin Key Laboratory of Pulp \& Paper, Tianjin University of Science \& Technology, Tianjin 300457, China \\ ${ }^{3}$ Engineering Research Center for Eco-Dyeing \& Finishing of Textiles, Ministry of Education, Zhejiang Sci-Tech University, \\ Hangzhou 310018, China
}

Correspondence should be addressed to Yanjun Tang; tangyj@zstu.edu.cn

Received 13 May 2013; Accepted 24 September 2013

Academic Editor: John Zhanhu Guo

Copyright (C) 2013 Yanjun Tang et al. This is an open access article distributed under the Creative Commons Attribution License, which permits unrestricted use, distribution, and reproduction in any medium, provided the original work is properly cited.

Novel polyvinyl alcohol (PVA)/styrene butadiene rubber (SBR) latex/carboxymethyl cellulose (CMC)/halloysite nanotubes (HNTs) nanocomposites were successfully prepared through physical blending. The as-obtained PVA/SBR/CMC/HNTs nanocomposites were coated on the surface of old corrugated container (OCC)-based paper in an effort to improve the mechanical properties of paper. To improve the dispersion of HNTs and enhance the compatibility between HNTs and polymer matrix, HNTs were modified with titanate coupling agent (TCA). FT-IR, together with TGA, confirmed that TCA was grafted onto the surface of HNTs successfully. XRD demonstrated that the crystal structures of HNTs remained almost unchanged. TEM showed that modified HNTs exhibited good dispersion and possessed nanotubular structures with an outer diameter of around $50 \mathrm{~nm}$ and an inner diameter of about $20 \mathrm{~nm}$. SEM gave an indication that modified HNTs were dispersed more uniformly than unmodified HNTs within PVA/SBR/CMC matrix. Rheological measurement exhibited that surface modification process enhanced the compatibility between HNTs and polymer matrix, thus resulting in the decreased viscosity of nanocomposites. In comparison with unmodified HNTs, modified HNTs were found to contribute more to the enhancement in mechanical properties, which might be attributed to the better dispersion and compatibility of modified HNTs evidenced by TEM, SEM, and rheological measurement.

\section{Introduction}

HNTs, derived from mineral resources deposited in such countries as China, America, New Zealand, France, and Belgium $[1,2]$, are naturally occurring aluminosilicate minerals with molecular formula of $\mathrm{Al}_{2} \mathrm{Si}_{2} \mathrm{O}_{5}(\mathrm{OH})_{4} \cdot \mathrm{nH}_{2} \mathrm{O}$ [3]. In general, HNTs, exhibiting multiwalled nanotube structures [4], which essentially belong to kaolin varieties, with the lengths of $300-1500 \mathrm{~nm}$, internal diameters of $15-100 \mathrm{~nm}$, and external diameters of 40-120 nm [5, 6]. However, HNTs are to some extent different from kaolin [7]. HNTs possess two-layered aluminosilicate mineral structures, where the aluminol $(\mathrm{Al}-\mathrm{OH})$ groups dominate the internal surface and siloxane ( $\mathrm{Si}-\mathrm{O}-\mathrm{Si})$ groups take up the outer surface [8-11]. Furthermore, as an alternative for carbon nanotubes, HNTs have their outstanding advantages of abundantly available resource and low cost [12-15]. As a consequence, HNTs have received much attention over the past decade in such fields as biomaterials [16] and nanocomposites [17]. There are many studies regarding the application of HNTs in improving the material properties in the previous literature. Ismail et al. [18] studied the morphological characteristics and mechanical properties of ethylene-propylene-diene monomer (EPDM) composites filled with HNTs and found that the tensile strength, stiffness, and ductility of EPDM/HNTs nanocomposites were simultaneously enhanced with the added HNTs, 
particularly at a high HNTs loading. Schmitt et al. [19] used both modified and unmodified HNTs as nanofillers to plasticized starch polymeric matrix and investigated the structural, morphological, thermal, and mechanical properties of plasticized starch/HNTs nanocomposites. Rybiński et al. [20] and Du et al. [21] studied the thermal properties and flammability of different nanocomposites based on HNTs, respectively, and proposed that the nanofillers can increase the thermal stability and decrease the flammability of nanocomposites.

However, much like other nanofillers, the main difficulty in utilization of HNTs in nanocomposites arises from the agglomeration of HNTs and the poor interfacial compatibility between HNTs and polymer matrix [22]. To overcome these disadvantages, many efforts have been made to disperse HNTs uniformly in polymer matrix and to improve the interfacial compatibility between HNTs and polymer matrix. Jia et al. [23] used complex of resorcinol and hexamethylenetetramine $(\mathrm{RH})$ to modify HNTs and found that $\mathrm{RH}$ can not only facilitate the dispersion and orientation of HNTs in SBR matrix at nanometer scale but also enhance the interfacial combination between HNTs and rubber matrix. Joo et al. [24] modified the functional groups of HNTs from hydroxyl groups (HNTs-OH) to carboxylic acids (HNTs-COOH) to improve the dispersion of HNTs in acidic, basic, and neutral solutions. Prashantha et al. [25] conducted the modification of HNTs with quaternary ammonium salt and concluded that modified HNTs can lead to better performances than unmodified HNTs due to strong interfacial interaction between the polymer matrix and the nanotubes.

Paper quality is strongly dependent on the structural and chemical properties of paper surface, which is not only associated with the fibrous matrix but also related to the final surface treatment [26]. Surface treatments such as coating and sizing are widely applied in papermaking industry to improve surface strength. Conventional polymer composites, such as PVA, copolymers of styrene and maleic anhydride (SMA), SBR, polyurethane (PU), and polyacrylamide (PAM), are often employed to improve the properties of paper by forming a layer of polymer film on the whole paper sheet [27]. However, there are limited studies regarding the nanocomposites containing HNTs for the surface coating of paper in the previous publications. In the present work, novel nanocomposites were designed using a blend of PVA, SBR, and CMC, especially the incorporation of modified HNTs. To achieve desired dispersion of HNTs nanofillers and to yield a good compatibility between HNTs and the polymer matrix, HNTs were modified first by TCA. Subsequently, PVA/SBR/CMC/HNTs nanocomposites were successfully prepared through physical blending. In addition, the effects of unmodified and modified HNTs on the nanocomposite properties and the subsequent paper properties were also studied.

\section{Experimental}

2.1. Materials. HNTs used in this study were mined from Hubei Province, China. TCA, possessing a decomposition temperature of $210^{\circ} \mathrm{C}$, a density of $1.095 \mathrm{~g} / \mathrm{cm}^{3}$, and a refractive index of 1.446, was purchased from Hangzhou Jessica
Chemical Co., Ltd. SBR-latex with a solid content of $50 \%$ was supplied by BASF. CMC was provided by Shanghai Sinopharm Chemical Reagent Co., Ltd. PVA was supported by a paper mill in Hangzhou City of China. Anhydrous ethanol, serving as the dissolvent in the modification process of HNTs, was purchased from Hangzhou Gaojing Fine Chemical Industry Co., Ltd. The commercial base paper with a basic weight of about $130 \mathrm{~g} / \mathrm{m}^{2}$ was provided by a paper mill in Zhenjiang Province of China. It was derived from OCC pulp, a cheap and low-grade raw material.

2.2. Surface Modification of HNTs. Initially, $2 \mathrm{~g}$ of HNTs were dispersed in $40 \mathrm{~mL}$ of anhydrous ethanol upon ultrasonic treatment for $5 \mathrm{~min}$. TCA was dissolved in $10 \mathrm{~mL}$ of anhydrous ethanol at the ratio (TCA/HNTs) of 3.75\%, 7.5\%, and $15 \%$, respectively, and then was added into the solution of HNTs. The mixture was reacted at a constant temperature of $80^{\circ} \mathrm{C}$ for $6 \mathrm{~h}$ under the mechanical stirring $(550 \mathrm{rpm})$ in a three-necked round-bottomed flask. Finally, the reaction mixture was filtered and washed with anhydrous ethanol for several times, prior to the drying process in an oven at $60^{\circ} \mathrm{C}$ for $24 \mathrm{~h}$.

\subsection{Preparation of PVA/SBR/CMC/HNTs Nanocomposites.} In this process, the PVA/SBR/CMC/HNTs nanocomposites with a solid content of $10 \%$ were made. The added proportion of PVA:SBR was 3:1, and the weight ratio of CMC/HNTs was $2: 1$. Initially, $6.375 \mathrm{~g}$ of PVA was added into distilled water and heated to $90-95^{\circ} \mathrm{C}$ until PVA was completely dissolved. Subsequently, specified amount of SBR was slowly added into the resulting PVA solution, followed by the addition of $1.0 \mathrm{~g}$ of CMC. Lastly, HNTs (unmodified sample or modified sample treated with $3.75 \%$ TCA) were gradually added into the PVA/SBR/CMC nanocomposites. As a result, $\mathrm{PVA} / \mathrm{SBR} / \mathrm{CMC} / \mathrm{HNTs}$ and $\mathrm{PVA} / \mathrm{SBR} / \mathrm{CMC} / \mathrm{MHNTs}$ nanocomposites were obtained.

2.4. Preparation of Coated Paper. OCC-based paper was selected as the base paper for surface coating process. The surface coating process was completed on a laboratory scale ZAA 2300 multicoater (Zehntner, Switzerland). About $10 \mathrm{~mL}$ of obtained nanocomposites were loaded on the base paper and then spread over with the aid of a round steel bar. The coated paper was initially dried at ambient temperature and then dried at $100^{\circ} \mathrm{C}$ for $20 \mathrm{~min}$. The coat weight of the paper samples was about $3 \mathrm{~g} / \mathrm{m}^{2}$.

2.5. Characterization. Fourier transformed infrared (FT-IR) spectra of HNTs were conducted on Nicolet 5700 spectrometer (Thermo Fisher Scientific, USA). The thermal behavior of modified and unmodified HNTs was determined with Pyrisl TGA (PerKin Elmer, USA) under nitrogen atmosphere at a heating rate of $20^{\circ} \mathrm{C} / \mathrm{min}$. X-ray diffraction (XRD) was performed on a diffractometer (X'TRA-055, ARL, Switzerland) with a nickel-filter $\mathrm{Cu} \mathrm{K} \alpha(\lambda=0.154 \mathrm{~nm})$. The morphology analysis was carried out by transmission electron microscopy (TEM) on a JSM-2100 electron microscope (JEOL, Japan) at an accelerating voltage of $200 \mathrm{KV}$. The morphology of 


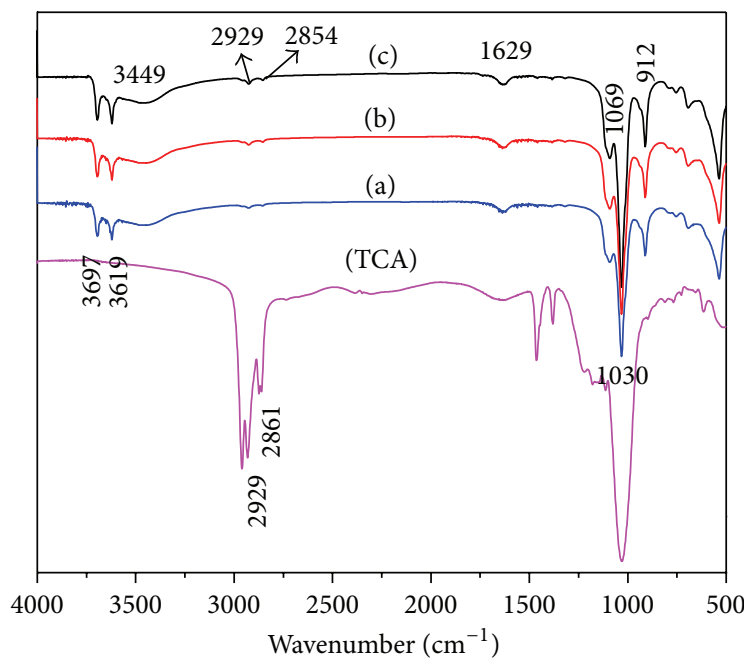

FIGURE 1: FT-IR spectra of pure TCA and HNTs modified with (a) $3.75 \%$, (b) $7.5 \%$, and (c) $15 \%$ of TCA.

the sized paper was observed by a ULTRA- 55 field emission scanning electron microscope (JEOL, Japan). Rheological behavior of the PVA/SBR/CMC/HNTs nanocomposites was determined by an advanced cylinder rotary rheometer Physica MCR301 (Anton Paar, Austria) at $25^{\circ} \mathrm{C}$. The mechanical strength of the paper coated with PVA/SBR/CMC/HNTs nanocomposites including tensile strength, tear strength, and burst strength was measured according to Chinese National Standards.

\section{Results and Discussion}

3.1. FT-IR Spectra. The FT-IR spectra of modified HNTs treated with various percentages of TCA were investigated, and the results are shown in Figure 1. For the sake of comparison, the spectrum of pure TCA is also presented. From the FT-IR spectra of modified HNTs (Figures 1(a), 1(b), and $1(\mathrm{c}))$, the absorbances at 3697,3619 , and $1629 \mathrm{~cm}^{-1}$ are associated with pure HNTs, mainly due to $\mathrm{O}-\mathrm{H}$ stretching of inner surface hydroxyl groups, $\mathrm{O}-\mathrm{H}$ stretching of inner hydroxyl groups, and decomposition of water, respectively [28]. Similarly, a very strong adsorption at $1030 \mathrm{~cm}^{-1}$ possibly originates from the asymmetric flexible vibration of $\mathrm{O}-\mathrm{Si}$ due to the plenty of $\mathrm{O}-\mathrm{Si}-\mathrm{O}$ groups on the outer surface. These characteristic absorption peaks mentioned above are close to the results reported in previous literature [29] for the same HNTs materials. In addition, it should be noted that the spectra of modified HNTs samples provide evidence of surface modification by the occurrence of the absorbance at $2929 \mathrm{~cm}^{-1}$ and $2854 \mathrm{~cm}^{-1}$. These bands are indicative of the stretching vibrations of $\mathrm{C}-\mathrm{H}$ bonds of TCA, which can be observed in the spectra of TCA shown in Figure 1. These changes in FT-IR spectra demonstrate that TCA is tightly absorbed on the surface of HNTs by chemisorptions, which is still existed on the surface of the modified particles even after being fully washed by anhydrous ethanol [30].

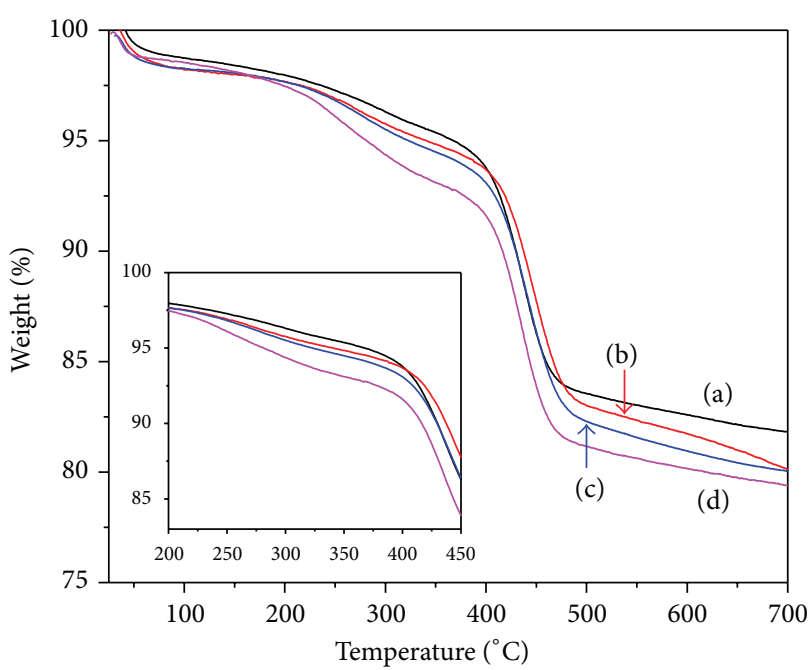

FIGURE 2: TGA curves of (a) unmodified HNTs and HNTs modified with (b) $3.75 \%$, (c) $7.5 \%$, (d) $15 \%$ of TCA.

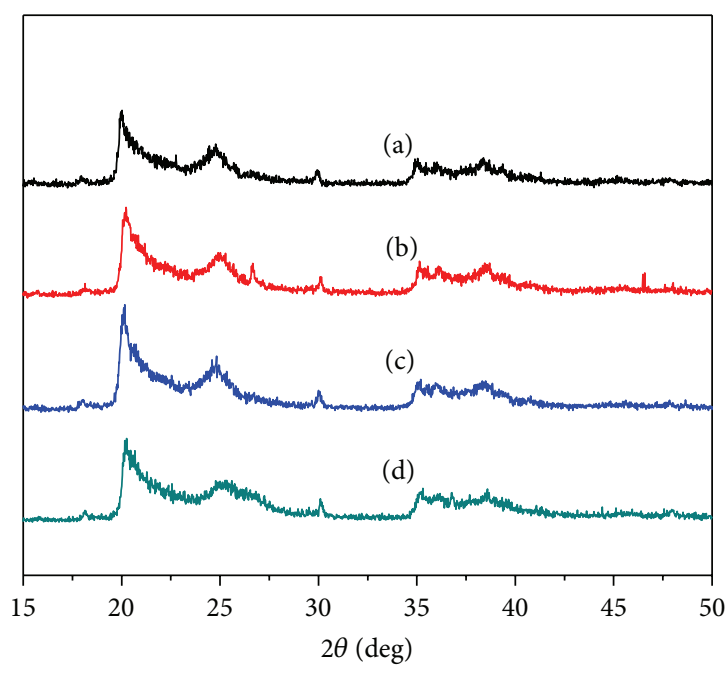

FIGURE 3: XRD patterns of (a) unmodified HNTs and HNTs modified with (b) $3.75 \%$, (c) $7.5 \%$, and (d) $15 \%$ of TCA.

3.2. Thermal Analysis. To figure out the TCA amount grafted onto the surface of HNTs, the unmodified and modified HNTs are characterized by TGA technique. Figure 2 shows the TGA curves of unmodified HNTs and modified HNTs with different percentages of TCA. As shown in Figure 2, the weight loss of unmodified HNTs is about $18 \%$ when it is heated from room temperature to $700^{\circ} \mathrm{C}$ (Figure 2(a)), which is probably attributed to the weight loss of hydroxyl groups and adsorbed matters on HNTs. The results are in good agreement with those of Zhang's investigation [31] where both the pure HNTs and PANI/HNTs nanocomposites exhibited a similar weight-loss trend, and the weight loss was less than $20 \%$. Moreover, the vast majority of the weight loss occurred in the range of $200-475^{\circ} \mathrm{C}$, which can make up the total weight above $60 \%$. In comparison with unmodified HNTs, the TGA curves (Figures 2(b), 2(c), and 2(d)) of modified HNTs with different percentages of TCA show 


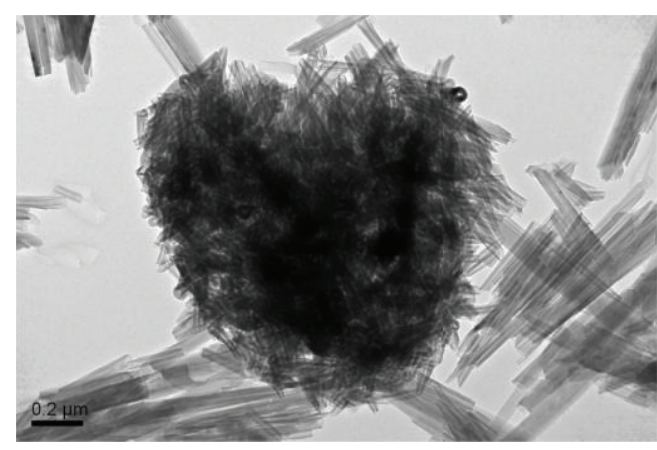

(a)

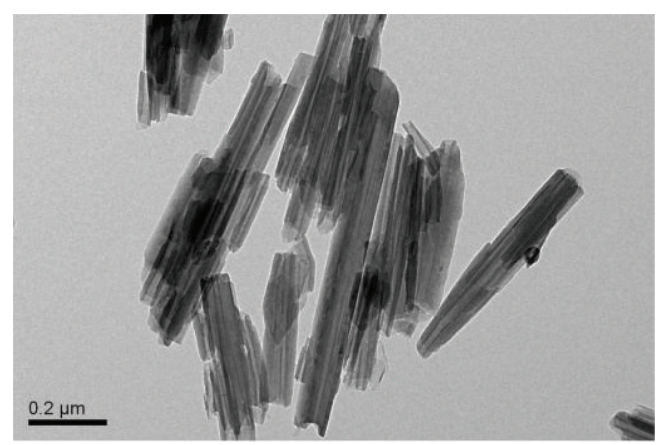

(c)

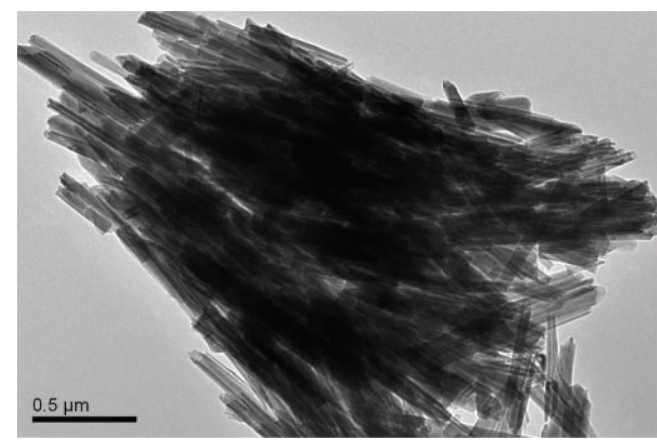

(b)

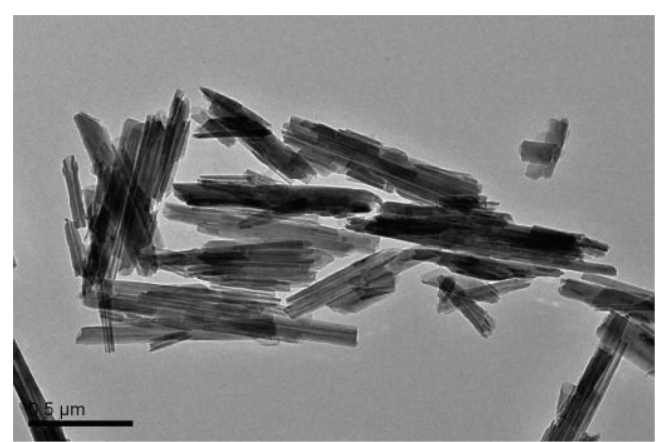

(d)

FIGURE 4: TEM images of (a), (b) unmodified HNTs and (c), (d) modified HNTs.

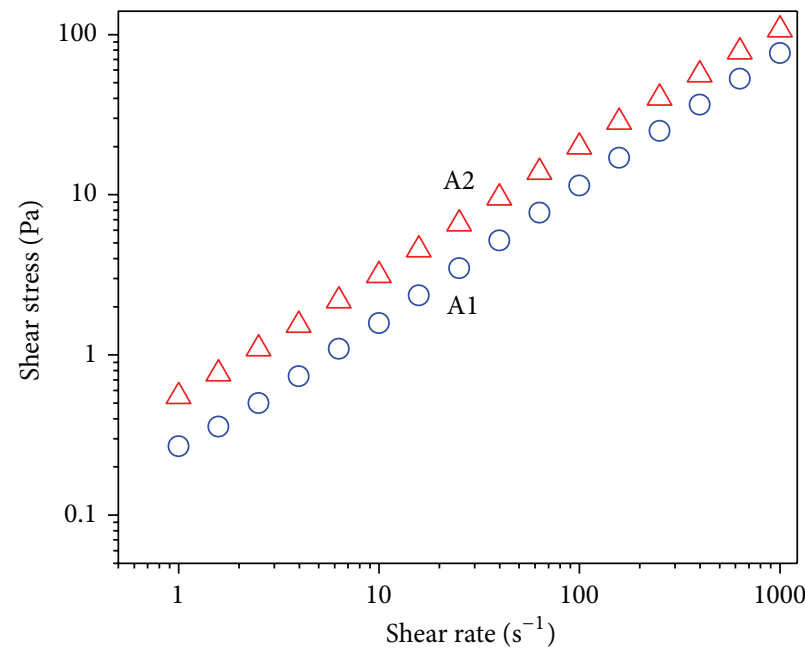

(a)

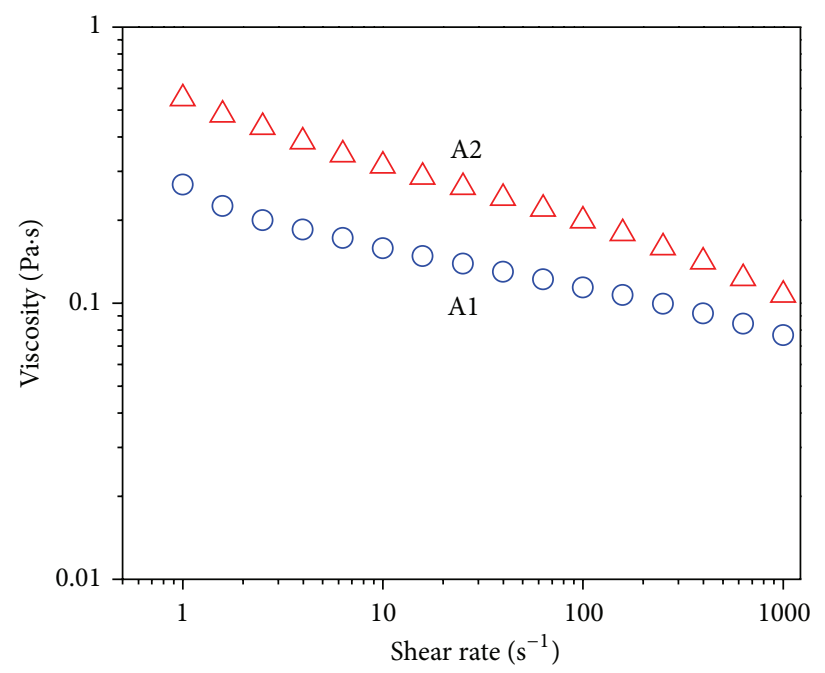

(b)

FIGURE 5: Shear stress (a) and shear viscosity (b) as a function of the shear rate for PVA/SBR/CMC nanocomposites with (A1) modified HNTs and (A2) unmodified HNTs.

a high decomposition rate with the ascent of temperature from 200 to $450^{\circ} \mathrm{C}$ due to the TCA grafting on HNTs. In addition, it can be derived from Figure 2 that the total weight loss of each modified HNTs is essentially consistent with the percentage of TCA used in surface modification. The bigger the TCA percentage used in surface modification, the higher the total weight loss of the modified HNTs. Therefore, similar to the results of FT-IR spectra, TGA also confirms that TCA is really anchored or grafted on the surface of HNTs, regardless of the various grafting ratio.

3.3. X-Ray Diffraction Analysis. To survey the influence of surface modification on the crystal structures of HNTs, X-ray diffraction patterns of the unmodified and modified HNTs were conducted, and the results are given in Figure 3. It is apparent that the $\mathrm{X}$-ray diffraction peaks of the modified 


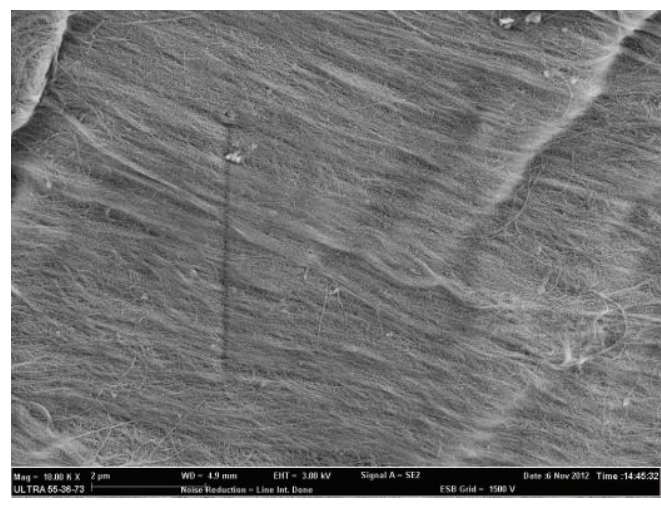

(a)

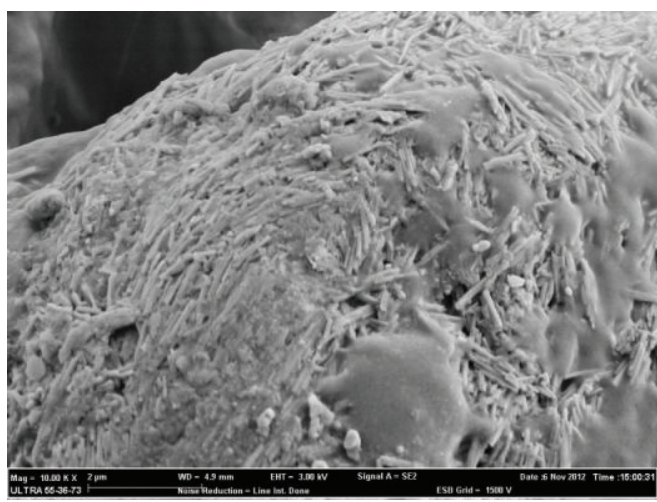

(c)

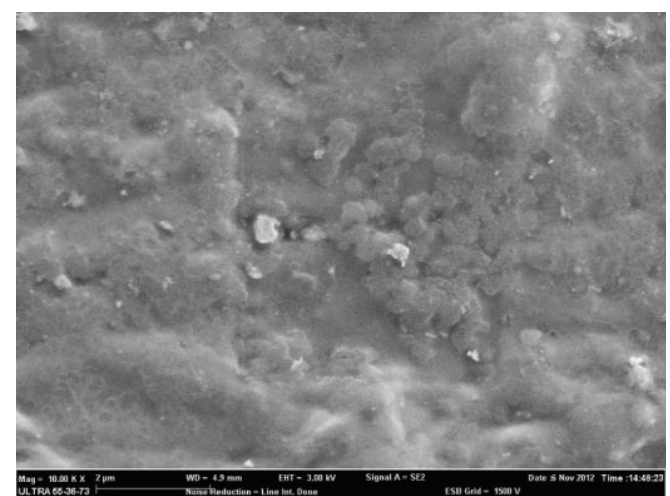

(b)

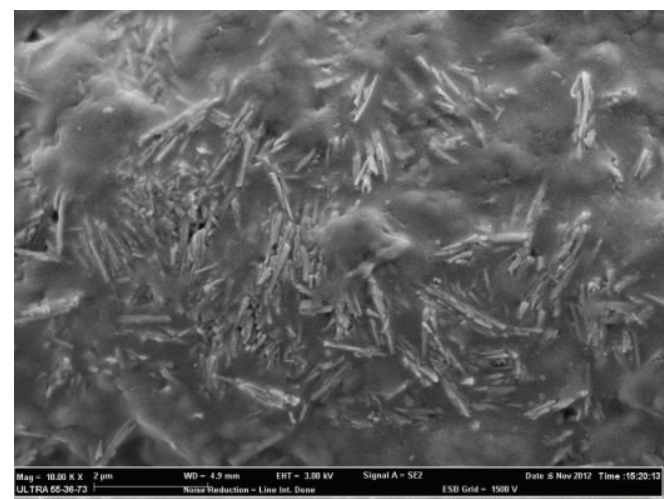

(d)

FIGURE 6: SEM images of (a) base paper, paper coated with (b) PVA/SBR composites, (c) PVA/SBR/CMC/HNTs nanocomposites, and (d) $\mathrm{PVA} / \mathrm{SBR} / \mathrm{CMC} / \mathrm{MHNTs}$ nanocomposites.

HNTs samples nearly remain consistent with the unmodified HNTs. After surface modification, there is no indication of the significant change in X-ray diffraction peaks (Figure 3), implying that the crystal structures of HNTs are not significantly altered, regardless of the surface modification.

3.4. TEM Analysis. TEM images of the unmodified and modified HNTs are illustrated in Figure 4. In order to obtain detailed morphological information of the specimens, different magnifications of various samples are presented. In Figures 4(a) and 4(b), an obvious agglomeration can be observed, indicating that the unmodified HNTs exhibit low polydispersity, which is certainly unfavorable to their reinforcement capability. However, in Figures 4(c) and 4(d), it can be observed that the modified HNTs are well separated and exhibit almost ideal nanotubular structures with an outer diameter of around $50 \mathrm{~nm}$, an inner diameter of about $20 \mathrm{~nm}$, and a length of about $1000 \mathrm{~nm}$. One can draw a conclusion that surface modification of HNTs nanofillers with TCA can effectively prevent their agglomeration and greatly improve the dispersion properties. The improvement in HNTs dispersion properties might be explained that TCA imparts nonpolar functional groups to HNTs, thus leading to the significant decrease in surface energy of HNTs.
3.5. Rheological Behavior of PVA/SBR/CMC/HNTs Nanocomposites. Rheological measurement is generally recognized as an indirect method to describe the dispersion state of particles in polymer matrix [32]. To investigate the compatibility between HNTs and the polymer matrix, the rheological behavior of PVA/SBR/CMC/HNTs nanocomposites was measured, and the rheological behavior curves are presented in Figures 5(a) and 5(b). As can be seen, the nanocomposites exhibit a nonlinear flow curve with shear-thinning behavior, providing direct evidence that PVA/SBR/CMC/HNTs nanocomposites are characteristics of a typical nonNewtonian fluid [33]. It is apparent in Figure 5(a) that the shear stress increases with increasing the shear rate, showing a behavior close to Bingham pseudoplastic fluids. In Figure 5(b), it can also be observed that the viscosity decreases as the shear rate increases, which may be due to the presence of random-oriented and highly entangled state of the PVA/SBR/CMC polymer chains under high shear rate [32]. More importantly, compared to PVA/SBR/CMC/HNTs nanocomposites, PVA/SBR/CMC/MHNTs nanocomposites are found to exhibit a marked decline in viscosity at the same shear rate. This finding could possibly be explained by virtue of the fact that TCA can react with hydroxyl groups at the HNTs surface, which facilitates the formation of monomolecular layer on the HNTs. Thus, the formed monomolecular 
TABLE 1: Effect of PVA/SBR/CMC/HNTs nanocomposites on the mechanical properties of paper.

\begin{tabular}{lccc}
\hline Sample & $\begin{array}{c}\text { Tensile index } \\
\left(\mathrm{N} \cdot \mathrm{m} \cdot \mathrm{g}^{-1}\right)\end{array}$ & $\begin{array}{c}\text { Tear index } \\
\left(\mathrm{mN} \cdot \mathrm{m}^{2} \cdot \mathrm{g}^{-1}\right)\end{array}$ & $\begin{array}{c}\text { Burst index } \\
\left(\mathrm{KPa} \cdot \mathrm{m}^{2} \cdot \mathrm{g}^{-1}\right)\end{array}$ \\
\hline Base paper & 22.23 & 4.97 & 1.69 \\
Coated paper with PVA/SBR/CMC/HNTs nanocomposites & 23.53 & 5.23 & 1.95 \\
Coated paper with PVA/SBR/CMC/MHNTs nanocomposites & 25.26 & 5.71 & 2.08 \\
\hline
\end{tabular}

layer may increase the compatibility and reduce the flow resistance between $\mathrm{HNTs}$ and PVA/SBR/CMC composites [34].

\subsection{Microstructure of Paper Coated with PVA/SBR/CMC/} HNTs Nanocomposites. It is well known that uniform dispersion of nanofillers in polymer matrix is a key factor affecting the improvement of the mechanical properties of polymer materials [35]. SEM images were employed to investigate the interaction between HNTs and polymer composites on the base paper. Figure 6(a) shows the SEM image of base paper surface. It can be perceived that the base paper contains interlaced cellulose fibrils and appears to have rough surfaces. The microstructure of sized paper with conventional PVA/SBR composites can be observed in Figure 6(b). It presents that the base paper is covered with polymer film except some uneven spacing. Moreover, the SEM images of paper sized with HNTs-polymer nanocomposites are shown in Figures 6(c) and 6(d). In comparison with Figure 6(c), Figure $6(\mathrm{~d})$ reveals that the majority of modified HNTs are embedded and uniformly dispersed in the polymer matrix coated on the base paper, which is likely due to the increased surface hydrophobicity and decreased surface free energy [33]. In addition, the interface between modified HNTs and PVA/SBR/CMC matrix is blurry and hardly debonded, suggesting the very strong interfacial bonding between modified HNTs and PVA/SBR/CMC matrix [29]. Similar dispersion behavior of HNTs in specific polymer matrix was reported in previous publications [36,37]. All of the above results further support that surface modification of HNTs with TCA has an important impact on the dispersion properties in polymer matrix, which would subsequently facilitate the potential application in surface coating of paper.

3.7. Mechanical Properties of Paper Coated with PVA/SBR/ CMC/HNTs Nanocomposites. The mechanical properties of base paper, as well as the paper coated with PVA/SBR/ $\mathrm{CMC} / \mathrm{HNTs}$ nanocomposites and PVA/SBR/CMC/MHNTs nanocomposites, are illustrated in Table 1 . The tensile index, tear index and burst index of the base paper are $22.23 \mathrm{~N} \cdot \mathrm{m} \cdot \mathrm{g}^{-1}, 4.97 \mathrm{mN} \cdot \mathrm{m}^{2} \cdot \mathrm{g}^{-1}$, and $1.69 \mathrm{KPa} \cdot \mathrm{m}^{2} \cdot \mathrm{g}^{-1}$, respectively. As expected, the incorporation of HNTs in nanocomposites indeed exerts an important impact on the mechanical strength of sized paper, which can be attributed to the nanotubular structure of HNTs with high surface area. More importantly, it can be derived that the application effect of HNTs in PVA/SBR/CMC nanocomposites is strongly associated with their surface chemistry and structure derived from surface modification process. As shown in Table 1 , compared with PVA/SBR/CMC/HNTs nanocomposites, PVA/SBR/CMC/MHNTs nanocomposites are found to make the coated paper remarkably increased by $7.35 \%, 9.18 \%$, and $6.67 \%$ in tensile index, tear index, and burst index, respectively. The significant reinforcing effects of PVA/SBR/CMC/MHNTs nanocomposites might be due to the uniform dispersion of modified HNTs and strong interfacial interaction assigned to hydrogen bonding between HNTs and the polymer matrix [34]. Consequently, all of these further indicate that surface modification of the HNTs is a key factor affecting the further application of HNTs in polymer matrix.

\section{Conclusions}

In this work, novel PVA/SBR/CMC/HNTs nanocomposites were prepared for the potential application in surface coating of paper. To enhance the application effect of HNTs in polymer composites, the surface of HNTs was modified with TCA. The results showed that modified HNTs exhibited good dispersion and displayed an ideal interfacial compatibility within PVA/SBR/CMC composites. Compared to unmodified HNTs, the incorporation of modified HNTs in PVA/SBR/CMC nanocomposites showed more significant improvement in tensile index, tear index, and burst index of coated paper, which is mainly due to the better dispersion and interfacial compatibility.

\section{Acknowledgments}

The research is grateful for the financial support from the National Natural Science Foundation of China (Grant no. 31100442), Foundation (no. 201108) of Tianjin Key Laboratory of Pulp \& Paper (Tianjin University of Science \& Technology, China), the Science and Technology Program of Zhejiang Environmental Protection Bureau of China (Grant no. 2012B008), and 521 Talent Cultivation Program of Zhejiang Sci-Tech University (Grant no. 11110132521310).

\section{References}

[1] B. Lecouvet, J. G. Gutierrez, M. Sclavons, and C. Bailly, "Structure-property relationships in polyamide 12/halloysite nanotube nanocomposites," Polymer Degradation and Stability, vol. 96, no. 2, pp. 226-235, 2011.

[2] M. Zhao and P. Liu, "Adsorption behavior of methylene blue on halloysite nanotubes," Microporous and Mesoporous Materials, vol. 112, no. 1-3, pp. 419-424, 2008.

[3] X. M. Sun, Y. Zhang, H. B. Shen, and N. Q. Jia, "Direct electrochemistry and electrocatalysis of horseradish peroxidase 
based on halloysite nanotubes/chitosan nanocomposite film," Electrochimica Acta, vol. 56, no. 2, pp. 700-705, 2010.

[4] Y. Lin, K. M. Ng, C.-M. Chan, G. Sun, and J. Wu, "High-impact polystyrene/halloysite nanocomposites prepared by emulsion polymerization using sodium dodecyl sulfate as surfactant," Journal of Colloid and Interface Science, vol. 358, no. 2, pp. 423429, 2011.

[5] M. X. Liu, B. C. Guo, M. L. Du, F. Chen, and D. M. Jia, "Halloysite nanotubes as a novel $\beta$-nucleating agent for isotactic polypropylene," Polymer, vol. 50, no. 13, pp. 3022-3030, 2009.

[6] K. Prashantha, H. Schmitt, M. F. Lacrampe, and P. Krawczak, "Mechanical behaviour and essential work of fracture of halloysite nanotubes filled polyamide 6 nanocomposites," Composites Science and Technology, vol. 71, no. 16, pp. 1859-1866, 2011.

[7] E. E. Ibrahim, D. M. Chipara, R. Thapa, K. Lozano, and M. Chipara, "Raman spectroscopy of isotactic polypropylenehalloysite nanocomposites," Journal of Nanomaterials, vol. 2012, Article ID 793084, 8 pages, 2012.

[8] S. A. Hashemifard, A. F. Ismail, and T. Matsuura, "Mixed matrix membrane incorporated with large pore size halloysite nanotubes (HNTs) as filler for gas separation: morphological diagram," Chemical Engineering Journal, vol. 172, no. 1, pp. 581590, 2011.

[9] W. N. Xing, L. Ni, P. W. Huo et al., "Preparation high photocatalytic activity of CdS/halloysite nanotubes (HNTs) nanocomposites with hydrothermal method," Applied Surface Science, vol. 259, pp. 698-704, 2012.

[10] E. Tierrablanca, J. Romero-García, P. Roman, and R. CruzSilva, "Biomimetic polymerization of aniline using hematin supported on halloysite nanotubes," Applied Catalysis A, vol. 381, no. 1-2, pp. 267-273, 2010.

[11] M. X. Liu, W. D. Li, J. H. Rong, and C. R. Zhou, "Novel polymer nanocomposite hydrogel with natural clay nanotubes," Colloid and Polymer Science, vol. 290, no. 10, pp. 895-905, 2012.

[12] R. C. Liu, B. Zhang, D. D. Mei, H. Q. Zhang, and J. D. Liu, "Adsorption of methyl violet from aqueous solution by halloysite nanotubes," Desalination, vol. 268, no. 1-3, pp. 111-116, 2011.

[13] P. Luo, Y. Zhao, B. Zhang, J. Liu, Y. Yang, and J. Liu, "Study on the adsorption of Neutral Red from aqueous solution onto halloysite nanotubes," Water Research, vol. 44, no. 5, pp. 14891497, 2010.

[14] S. Q. Deng, J. N. Zhang, and L. Ye, "Halloysite-epoxy nanocomposites with improved particle dispersion through ball mill homogenisation and chemical treatments," Composites Science and Technology, vol. 69, no. 14, pp. 2497-2505, 2009.

[15] B. C. Guo, F. Chen, Y. D. Lei, X. L. Liu, J. J. Wan, and D. M. Jia, "Styrene-butadiene rubber/halloysite nanotubes nanocomposites modified by sorbic acid," Applied Surface Science, vol. 255, no. 16, pp. 7329-7336, 2009.

[16] M. X. Liu, Y. Zhang, C. C. Wu, S. Xiong, and C. R. Zhou, "Chitosan/halloysite nanotubes bionanocomposites: structure, mechanical properties and biocompatibility," International Journal of Biological Macromolecules, vol. 51, no. 4, pp. 566-575, 2012.

[17] J. M. Duan, R. C. Liu, T. Chen, B. Zhang, and J. D. Liu, "Halloysite nanotube- $\mathrm{Fe}_{3} \mathrm{O}_{4}$ composite for removal of methyl violet from aqueous solutions," Desalination, vol. 293, pp. 4652, 2012.

[18] H. Ismail, P. Pasbakhsh, M. N. A. Fauzi, and A. Abu Bakar, "Morphological, thermal and tensile properties of halloysite nanotubes filled ethylene propylene diene monomer (EPDM) nanocomposites," Polymer Testing, vol. 27, no. 7, pp. 841-850, 2008.

[19] H. Schmitt, K. Prashantha, J. Soulestin, M. F. Lacrampe, and P. Krawczak, "Preparation and properties of novel melt-blended halloysite nanotubes/wheat starch nanocomposites," Carbohydrate Polymers, vol. 89, no. 3, pp. 920-927, 2012.

[20] P. Rybiński and G. Janowska, “Thermal properties and flammability of nanocomposites based on nitrile rubbers and activated halloysite nanotubes and carbon nanofibers," Thermochimica Acta, vol. 549, pp. 6-12, 2012.

[21] M. Du, B. Guo, and D. Jia, "Thermal stability and flame retardant effects of halloysite nanotubes on poly(propylene)," European Polymer Journal, vol. 42, no. 6, pp. 1362-1369, 2006.

[22] L. C. Tan, Y. W. Chen, W. H. Zhou, and S. W. Ye, "Crystallization behavior and mechanical strength of poly (butylene succinateco-ethylene glycol)-based nanocomposites using functionalized multiwalled carbon nanotubes," Polymer Engineering and Science, vol. 52, no. 12, pp. 2506-2517, 2012.

[23] Z.-X. Jia, Y.-F. Luo, S.-Y. Yang, B.-C. Guo, M.-L. Du, and D.-M. Jia, "Morphology, interfacial interaction and properties of styrene-butadiene Rubber/Modified halloysite nanotube nanocomposites," Chinese Journal of Polymer Science, vol. 27, no. 6, pp. 857-864, 2009.

[24] Y. Joo, Y. Jeon, S. U. Lee et al., "Aggregation and stabilization of carboxylic acid functionalized halloysite nanotubes (HNTCOOH)," Journal of Physical Chemistry C, vol. 116, no. 34, pp. 18230-18235, 2012.

[25] K. Prashantha, M. F. Lacrampe, and P. Krawczak, "Processing and characterization of halloysite nanotubes filled polypropylene nanocomposites based on a masterbatch route: effect of halloysites treatment on structural and mechanical properties," Express Polymer Letters, vol. 5, no. 4, pp. 295-307, 2011.

[26] T. Enomae, N. Yamaguchi, and F. Onabe, "Influence of coating properties on paper-to-paper friction of coated paper," Journal of Wood Science, vol. 52, no. 6, pp. 509-513, 2006.

[27] A. Ashori, W. D. Raverty, and J. Harun, "Effect of chitosan addition on the surface properties of kenaf (Hibiscus cannabinus) paper," Fibers and Polymers, vol. 6, no. 2, pp. 174-179, 2005.

[28] M. Du, B. Guo, M. Liu, X. Cai, and D. Jia, "Reinforcing thermoplastics with hydrogen bonding bridged inorganics," Physica B, vol. 405, no. 2, pp. 655-662, 2010.

[29] M. L. Du, B. C. Guo, Y. D. Lei, M. X. Liu, and D. M. Jia, "Carboxylated butadiene-styrene rubber/halloysite nanotube nanocomposites: interfacial interaction and performance," Polymer, vol. 49, no. 22, pp. 4871-4876, 2008.

[30] Y. L. Tai, J. S. Qian, Y. C. Zhang, and J. D. Huang, "Study of surface modification of nano- $\mathrm{SiO}_{2}$ with macromolecular coupling agent (LMPB-g-MAH)," Chemical Engineering Journal, vol. 141, no. 1-3, pp. 354-361, 2008.

[31] W. L. Zhang and H. J. Choi, "Fabrication of semiconducting polyaniline-wrapped halloysite nanotube composite and its electrorheology," Colloid and Polymer Science, vol. 290, no. 17, pp. 1743-1748, 2012.

[32] C. Y. Tang, T. M. Yue, D. Z. Chen, and C. P. Tsui, "Effect of surface coating on the rheological properties of a highly opaque nano- $\mathrm{TiO}_{2} / \mathrm{HIPS}$ composite," Materials Letters, vol. 61, no. 2324, pp. 4618-4621, 2007.

[33] Z. Y. Yang, Y. J. Tang, and J. H. Zhang, "Surface modification of $\mathrm{CaCO}_{3}$ nanoparticles with silane coupling agent for improvement of the interfacial compatibility with styrene-butadiene 
rubber(SBR) latex," Chalcogenide Letters, vol. 10, no. 4, pp. 131141, 2013.

[34] C. Ai Wah, L. Yub Choong, and G. Seng Neon, "Effects of titanate coupling agent on rheological behaviour, dispersion characteristics and mechanical properties of talc filled polypropylene," European Polymer Journal, vol. 36, no. 4, pp. 789-801, 2000.

[35] J. H. Gou, S. O'Braint, H. Gu, and G. Song, "Damping augmentation of nanocomposites using carbon nanofiber paper," Journal of Nanomaterials, vol. 2006, Article ID 32803, 7 pages, 2006.

[36] N.-Y. Ning, Q.-J. Yin, F. Luo, Q. Zhang, R. Du, and Q. $\mathrm{Fu}$, "Crystallization behavior and mechanical properties of polypropylene/halloysite composites," Polymer, vol. 48, no. 25, pp. 7374-7384, 2007.

[37] S. Rooj, A. Das, V. Thakur, R. N. Mahaling, A. K. Bhowmick, and G. Heinrich, "Preparation and properties of natural nanocomposites based on natural rubber and naturally occurring halloysite nanotubes," Materials and Design, vol. 31, no. 4, pp. 21512156, 2010. 

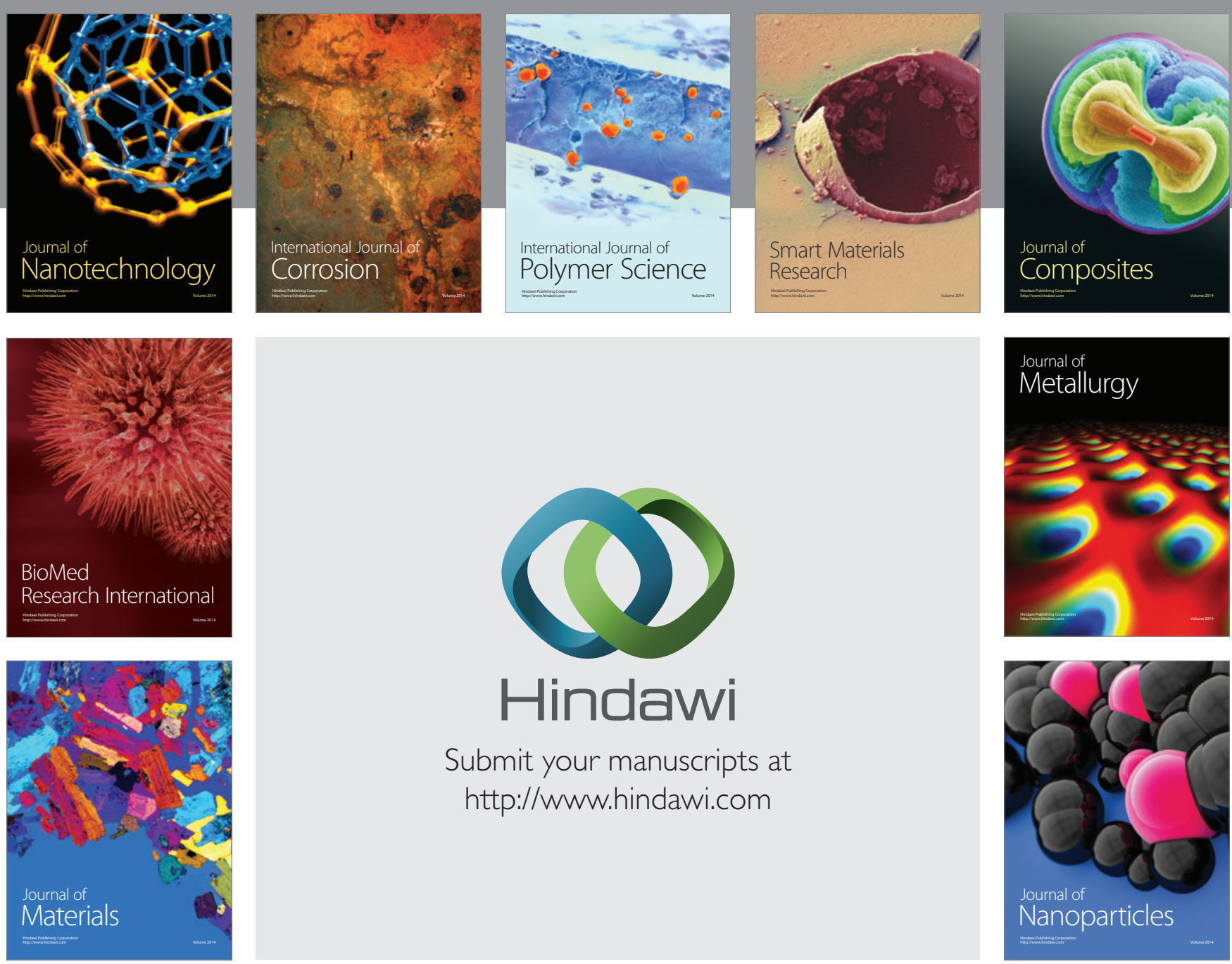

Submit your manuscripts at http://www.hindawi.com
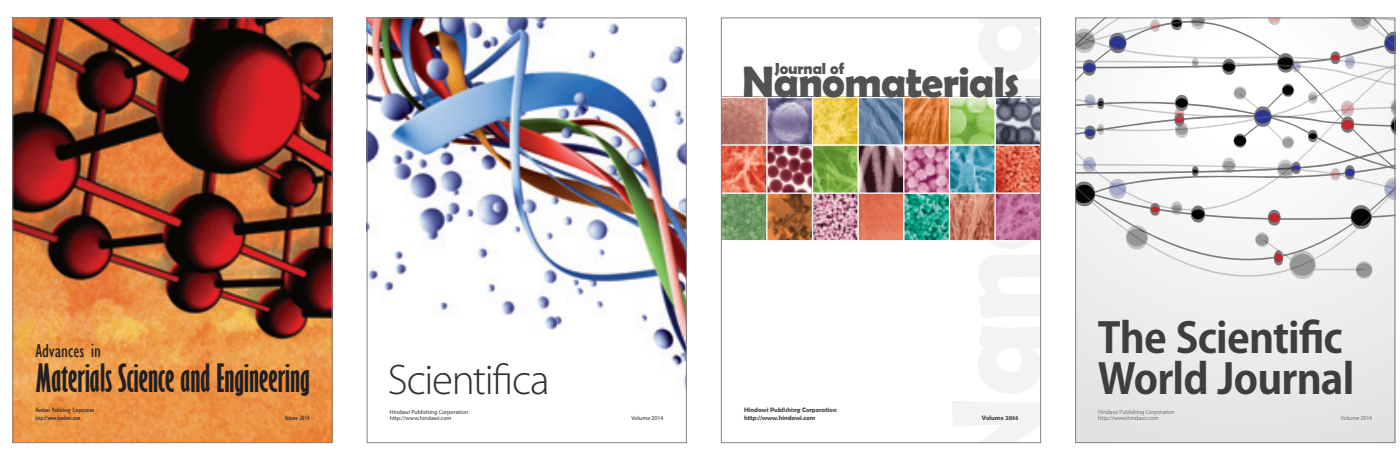

\section{The Scientific World Journal}
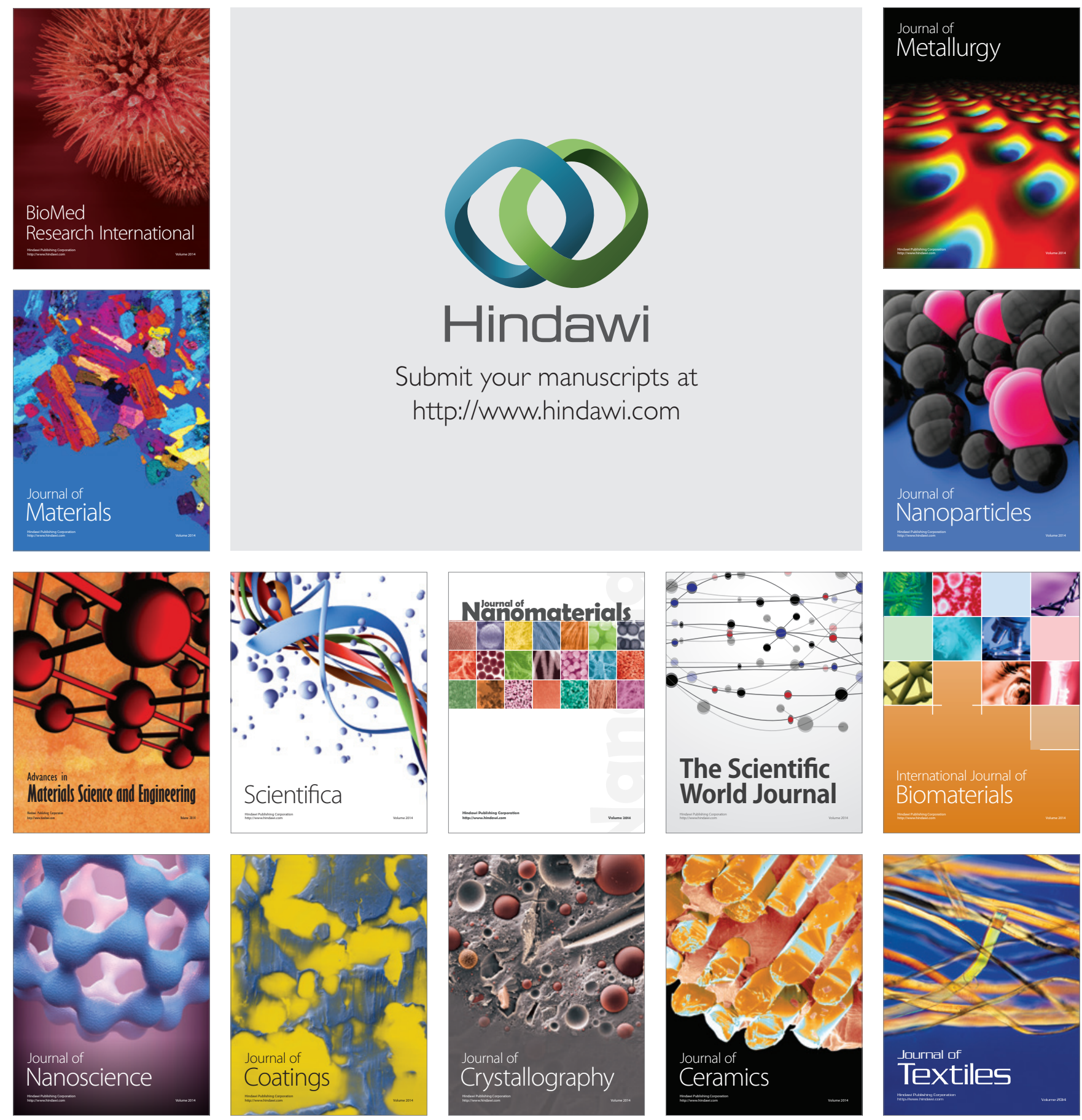